NISSUNA UMANA INVESTIGAZIONE SI PUO DIMANDARE VERA SCIENZIA S'ESSA NON PASSA PER LE MATEMATICHE DIMOSTRAZIONI LEONARDO DA VINCI

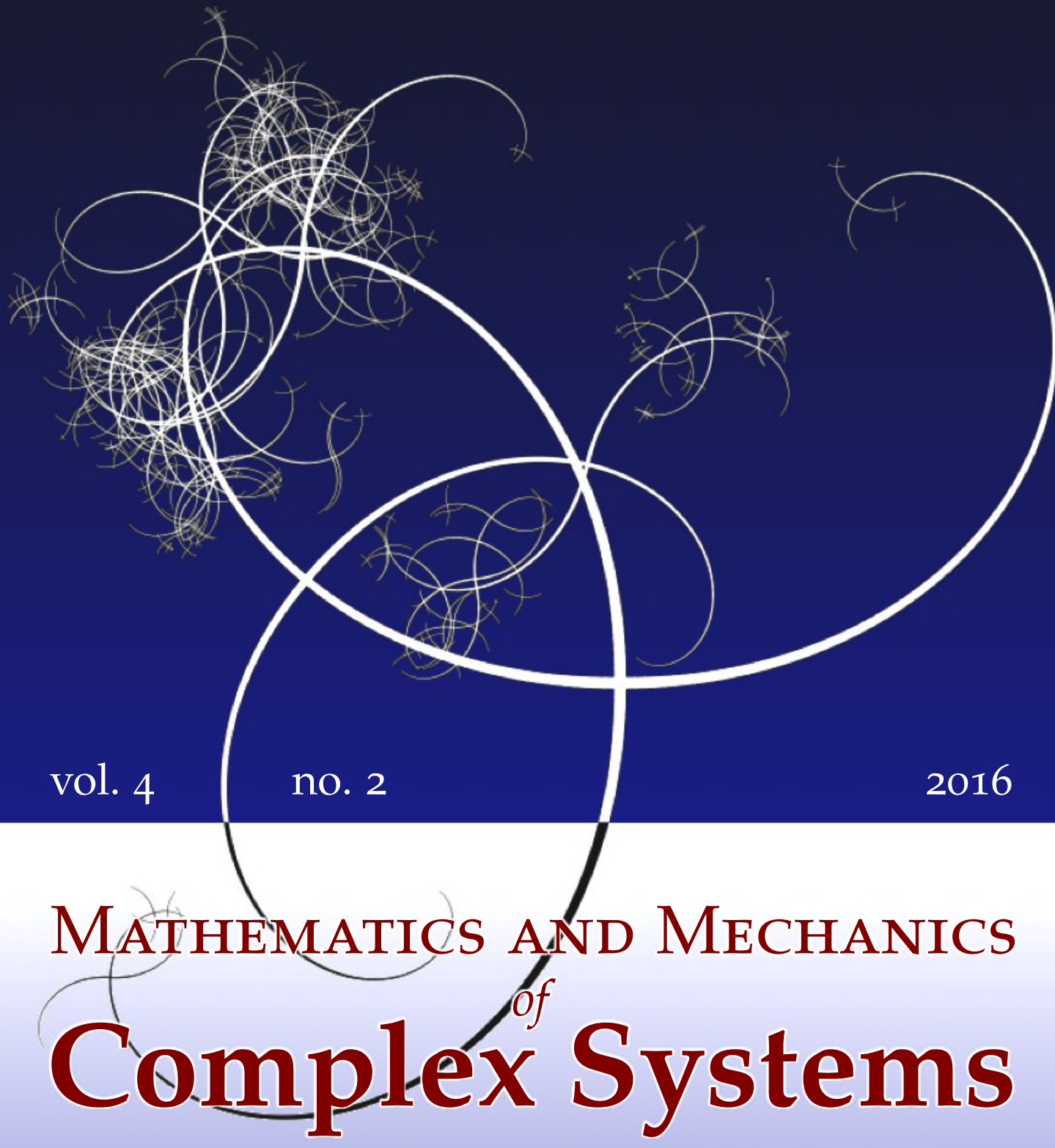

FABIo MANGINI AND FABRIZIO FREZZA

ANALYSIS OF THE ELECTROMAGNETIC REFLECTION AND TRANSMISSION THROUGH A STRATIFIED LOSSY MEDIUM OF AN ELLIPTICALLY POLARIZED PLANE WAVE 


\title{
ANALYSIS OF THE ELECTROMAGNETIC REFLECTION AND TRANSMISSION THROUGH A STRATIFIED LOSSY MEDIUM OF AN ELLIPTICALLY POLARIZED PLANE WAVE
}

\author{
FABIo MANGINi AND FABRIZIO FREZZA
}

\begin{abstract}
In this paper, a method to analyze the electromagnetic scattering of an elliptically polarized plane wave through a stratified lossy medium is presented. The interaction of the electromagnetic radiation with the stratified material is taken into account by means of the transfer-matrix approach: in this way, we can consider the stratified medium as an effective single interface. To do that, it was necessary to represent the complex plane-wave propagation vector with two different formulations: the phase and attenuation vectors and the complex angle. Thanks to these two formalisms, it is possible to describe the behaviors of this canonical phenomenon in an elegant way in all the cases of presence of a stratified lossy medium. A numerical code has been implemented to compute the field over the whole space. Finally, to validate the presented model, comparisons with the results presented in the literature have been provided.
\end{abstract}

\section{Introduction}

The determination of the Fresnel coefficients due to the presence of a layered medium with plane interfaces has been the subject of several studies in the last decades because of its important applications, e.g., to forward and inverse scattering by buried two- and three-dimensional objects, to electromagnetic artificial materials, to characterization of porous media and to enhancement of optical antennas and photovoltaic panels [Takenaka et al. 2003; Khoo et al. 2006; Feng et al. 2003; Ziolkowski and Erentok 2006; Taminiau et al. 2008; Frezza et al. 2013; 2015]. We consider the propagation of an inhomogeneous plane wave in a lossy medium, which impinges on the interface with another lossy medium. We assume the media are linear, isotropic, homogeneous, dielectric and generally dispersive and dissipative; with these exceptions, we define the problem in the most general manner. In general, from an electromagnetic point of view, all the materials can be subdivided into three categories: the vacuum with null conductivity $(\sigma=0)$,

\section{Communicated by Francesco dell'Isola.}

MSC2010: 78A25, 78A48.

Keywords: lossy media, transmission and reflection, stratified medium. 


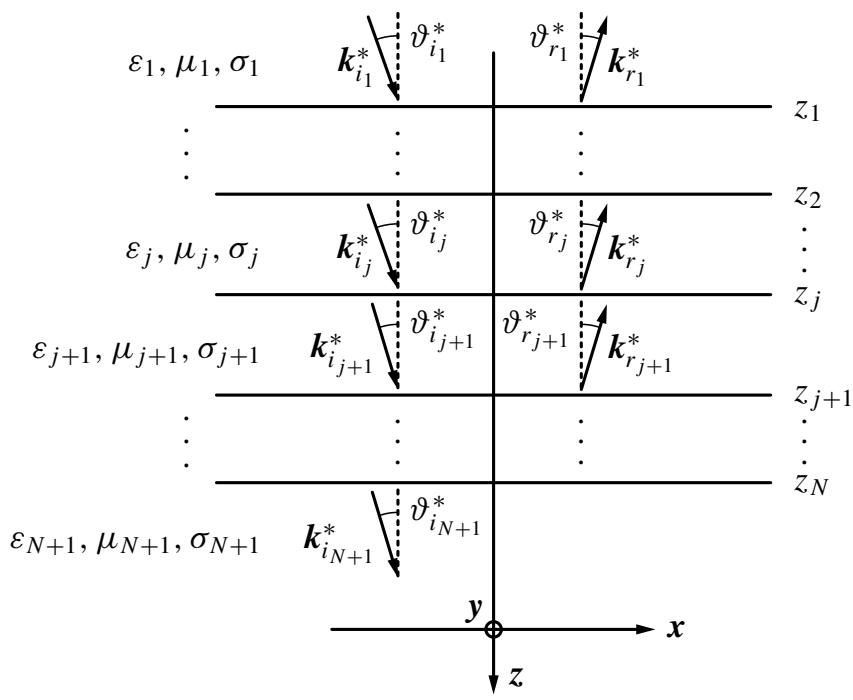

Figure 1. Geometry of the problem with the complex-angle formulation.

absolute dielectric permittivity $\varepsilon=\varepsilon_{0}$ and absolute permeability $\mu=\mu_{0}$; the lossless dielectrics with $\sigma=0, \varepsilon=\varepsilon_{0} \varepsilon_{r}$ and $\mu=\mu_{0} \mu_{r}$; the lossy medium with $\sigma \neq 0$, $\varepsilon=\varepsilon_{0} \varepsilon_{r}$ and $\mu=\mu_{0} \mu_{r}$; and finally the perfect conductor with $\sigma \simeq \infty$. Now we can see the lossy media are the most general materials considerable. In this paper, the complex plane-wave propagation vectors are represented with different formulations: the complex-angle formulation [Ivlev 1987] (Figure 1) and the phase and attenuation vectors, i.e., the Adler-Chu-Fano formulation [Adler et al. 1960] (Figure 2). In Figure 1, considering the $j$-th layer, the impinging complex wave vectors $\boldsymbol{k}_{j}^{*}$ and the transmitted one, $\boldsymbol{k}_{j+1}^{*}$, are shown. The angles that these vectors form with the normal direction (z-axis) to the interfaces are $\vartheta_{j}^{*}$ and $\vartheta_{j+1}^{*}$, respectively. On the other hand, in Figure 2, the phase and attenuation vectors of the incident, $\boldsymbol{\beta}_{j}, \boldsymbol{\alpha}_{j}$, and of the transmitted, $\boldsymbol{\beta}_{j+1}, \boldsymbol{\alpha}_{j+1}$, waves are shown. The angles that these vectors form with the normal direction to the interface are, respectively, $\xi_{j}, \eta_{j}$ and $\xi_{j+1}, \eta_{j+1}$. Moreover, we define the angles between the phase and the attenuation vectors, in the two media, as $\zeta_{j}=\eta_{j}-\xi_{j}$ and $\zeta_{j+1}=\eta_{j+1}-\xi_{j+1}$, respectively. The difference between the complex angle of the transmitted wave vector and the angle of the transmitted phase vector is well understood. However, some confusion between these angles may occur. To emphasize the possible mistake that can be made between these formulations, we consider the arguments in [Canning 2011], where the expressions of the Fresnel coefficients found in the literature are questioned. The mistake has been pointed out and corrected in [Besieris 2011] but allows us to clarify the differences between the possible representations of the 


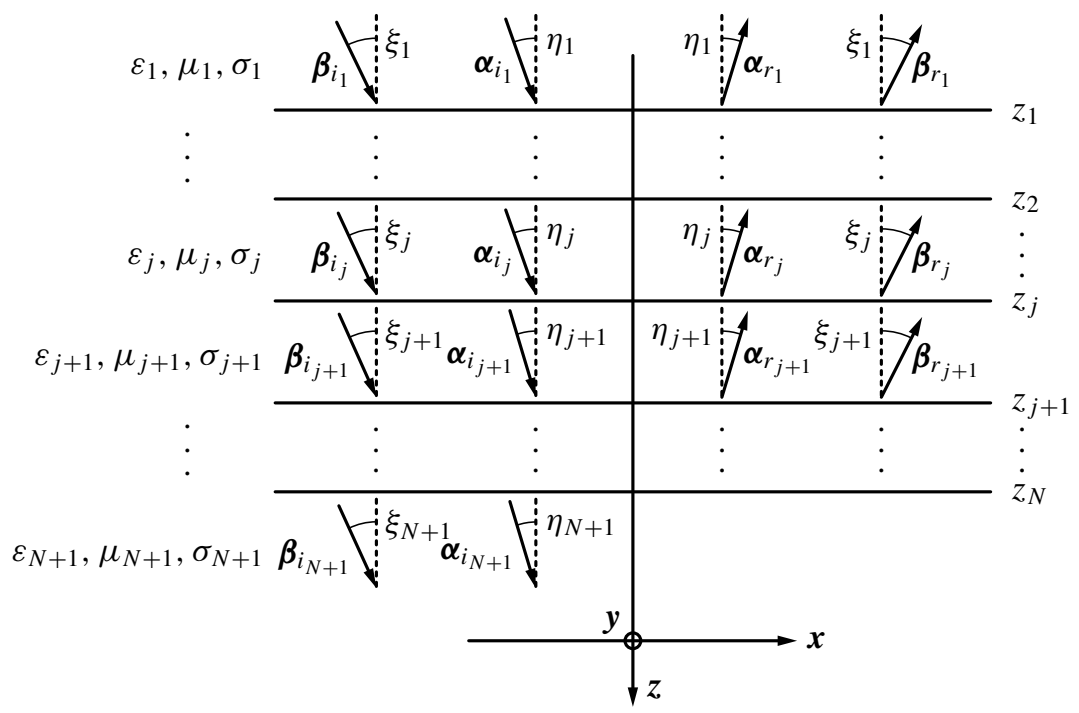

Figure 2. Geometry of the problem with the phase and attenuation vector representation.

complex wave vector of an inhomogeneous plane wave in a lossy medium. Moreover, the connection between these two formulations has been mentioned before, in [Roy 2003], where a numerical result, which needs different determinations in the solution of a polynomial equation of the fourth order and the inversion of a cosine function, is presented.

The two possible representations of a complex wave vector in a lossy medium are very useful to describe the most canonical scenarios about the stratified lossy medium. The complex-angle formulation can be the best solution to represent the transmission wave through a multilayered medium that will impinge on a buried sphere. The actual model of the complex wave vector consists of expanding in two different plane waves and then obtaining a single expansion involving the Wigner 3-j symbols [Kaplan and Resnikoff 1967], i.e., using the Adler-Chu-Fano formulation. Using the complex-angle formulation, it is possible to obtain an expansion of inhomogeneous elliptically polarized plane waves in terms of vectorial spherical harmonics in Mie form just using the Legendre functions generalized via hypergeometrical functions, instead of the classical Legendre functions. Hence, in all cases where a scatterer is present below the stratified lossy medium, in which the scattered electric field has to be represented as an expansion of vectorial harmonics, for example when scatterers with spherical, cylindrical or ellipsoidal shape are present, it is better to use the complex-angle formulation. Indeed, the phase and attenuation vector formulation is indispensable to obtain an elegant model of the classical generalized Fresnel problem. 
In this paper, we consider an elliptically polarized plane wave incident on the stratified material, we show the two possible representations of a complex wave vector in a lossy medium and we use the polarization vectors to calculate the real and imaginary parts of the complex angle as functions of the phase and attenuation vectors. The transmission through the stratified medium is determined by the well known formalism of the transition matrix. This matrix was first introduced by Abelès and is presented in many textbooks of optics and electromagnetics [Abelès 1950; Born and Wolf 1999; Chew 1995]. In the literature, many works have generalized the transmission-matrix method, e.g., with a polynomial expression of its elements [Vigoureux 1991] or by considering anisotropic layers [Essinger-Hileman 2013]. Thanks to the transfer-matrix formalism, the lossy medium stratification can be taken into account, for what concerns our problem, as an effective single interface. In particular, contrary to what is present in the literature, we have obtained an elegant matrix formulation, simply using the combination of the complex-angle formalism and the transfer-matrix approach. Moreover, in that way, we highlight the physical meaning of each element in the transition matrix.

Thanks to the transfer-matrix approach, we can easily extend the number of layers to infinity so as to realize an intermediate layer with peculiar properties. In particular, it will be possible to take into account some materials characterized by a relative permittivity that varies, for example, linearly between two external layers.

In Section 2, the theoretical formulation of the problem is presented in detail. In Section 3, in order to prove the correctness of our method, we show some comparisons with the results presented in the literature. Finally, in Section 4, the conclusions are drawn.

\section{Theoretical Approach}

The geometry of the problem is depicted in Figures 1 and 2. The incident monochromatic radiation on the first surface of the medium is a plane wave traveling from medium 1 to medium $N+1$. Let us call $z$ the stratification direction. Considering the $j$-th interface, the wave vector of the impinging radiation forms an angle $\vartheta_{j}$ with the $z$-axis, and its projection on the interface forms an angle $\varphi_{j}$ with the $x$ axis. Let $\varepsilon_{j}, \mu_{j}$ and $\sigma_{j}$ be the relative permittivity, the relative permeability, and the electrical conductivity in the $j$-th layer of the stratified medium. We remember that all media are linear, isotropic, homogeneous and generally dispersive and dissipative. Then the constitutive equations are

$$
\begin{aligned}
& \boldsymbol{D}(\boldsymbol{r}, \omega)=\varepsilon_{0} \varepsilon_{r} \boldsymbol{E}(\boldsymbol{r}, \omega), \\
& \boldsymbol{B}(\boldsymbol{r}, \omega)=\mu_{0} \mu_{r} \boldsymbol{H}(\boldsymbol{r}, \omega), \\
& \boldsymbol{J}(\boldsymbol{r}, \omega)=\sigma \boldsymbol{E}(\boldsymbol{r}, \omega),
\end{aligned}
$$


where $\omega$ is the angular frequency of the incident field. Solving the Helmholtz equation for the electric field, we can write the incident elliptically polarized field as

$$
\boldsymbol{E}_{i_{1}}(\boldsymbol{r})=\left(E_{i_{1}}^{H} \boldsymbol{\vartheta}_{0 i}+E_{i_{1}}^{E} \boldsymbol{\varphi}_{0 i}\right) e^{i \boldsymbol{k}_{i} \cdot \boldsymbol{r}}
$$

with

$$
\begin{aligned}
\boldsymbol{k}_{i_{1}} & =k_{1}^{*}\left(\sin \vartheta_{i_{1}}^{*} \cos \varphi_{i_{1}} \boldsymbol{x}_{0}+\sin \vartheta_{i_{1}}^{*} \sin \varphi_{i_{1}} \boldsymbol{y}_{0}+\cos \vartheta_{i_{1}}^{*} z_{0}\right), \\
\boldsymbol{\vartheta}_{0 i} & =\cos \vartheta_{i_{1}}^{*} \cos \varphi_{i_{1}} \boldsymbol{x}_{0}+\cos \vartheta_{i_{1}}^{*} \sin \varphi_{i_{1}} \boldsymbol{y}_{0}-\sin \vartheta_{i_{1}}^{*} z_{0}, \\
\boldsymbol{\varphi}_{0 i} & =-\sin \varphi_{i_{1}} \boldsymbol{x}_{0}+\cos \varphi_{i_{1}} \boldsymbol{y}_{0}
\end{aligned}
$$

and with $\boldsymbol{x}_{0}, \boldsymbol{y}_{0}$ and $z_{0}$ the cartesian unit vectors. Throughout this paper, a time dependence $e^{-i \omega t}$ is assumed and always omitted. The reflected $\boldsymbol{E}_{r}(\boldsymbol{r})$ and transmitted $\boldsymbol{E}_{t}(\boldsymbol{r})$ waves by the stratified medium are given by

$$
\begin{aligned}
& \boldsymbol{E}_{r}(\boldsymbol{r})=\left(R_{E}^{H} E_{i_{1}}^{H} \boldsymbol{\vartheta}_{0 r}+R_{E}^{E} E_{i_{1}}^{E} \boldsymbol{\varphi}_{0 r}\right) e^{i \boldsymbol{k}_{r} \cdot \boldsymbol{r}}, \\
& \boldsymbol{E}_{t}(\boldsymbol{r})=\left(T_{E}^{H} E_{i_{1}}^{H} \boldsymbol{\vartheta}_{0 t}+T_{E}^{E} E_{i_{1}}^{E} \boldsymbol{\varphi}_{0 t}\right) e^{i \boldsymbol{k}_{t} \cdot \boldsymbol{r}},
\end{aligned}
$$

where $R_{E}^{H}, T_{E}^{H}$ and $R_{E}^{E}, T_{E}^{E}$ are the effective reflection and transmission coefficients of the stratified medium for parallel $(\mathrm{E})$ and perpendicular $(\mathrm{H})$ polarizations of the electric field, respectively, relevant to the effective interface between medium 1 and medium $N+1$, with the vectors $\boldsymbol{k}_{r}, \boldsymbol{k}_{t}, \boldsymbol{\vartheta}_{0 r}, \boldsymbol{\vartheta}_{0 t}, \boldsymbol{\varphi}_{0 r}$ and $\boldsymbol{\varphi}_{0 t}$ having expressions similar to (5)-(7). For the sake of brevity, we work on the plane $\varphi=0$; however, the following considerations can be easily extended for each plane $\varphi \neq 0$. In this case, the wave number and the complex angle in (5)-(7) can be written as

$$
\begin{aligned}
& k^{*}=k_{\boldsymbol{R}}+i k_{\boldsymbol{I}}, \\
& \vartheta^{*}=\vartheta_{\boldsymbol{R}}+i \vartheta_{\boldsymbol{I}}
\end{aligned}
$$

[Adler et al. 1960]; i.e., it is always possible to represent the wave number and the impinging angle as constituted by a real part and an imaginary part. At the same time, as we can see from Figure 2, the complex wave vector can be represented as

$$
\boldsymbol{k}_{i}=\boldsymbol{\beta}_{i}+i \boldsymbol{\alpha}_{i}
$$

with

$$
\begin{aligned}
\boldsymbol{\beta}_{i} & =\beta_{i}\left(\sin \xi_{i} \boldsymbol{x}_{0}+\cos \xi_{i} z_{0}\right), \\
\boldsymbol{\alpha}_{i} & =\alpha_{i}\left(\sin \eta_{i} \boldsymbol{x}_{0}+\cos \eta_{i} z_{0}\right)
\end{aligned}
$$

being the phase vector and the attenuation vector, respectively. Another representation useful for our treatment is

$$
\begin{aligned}
& \boldsymbol{k}_{i}=k_{i_{\tau}} \boldsymbol{x}_{0}+k_{i_{n}} z_{0}, \\
& \boldsymbol{k}_{r}=k_{r_{\tau}} \boldsymbol{x}_{0}+k_{r_{n}} z_{0}
\end{aligned}
$$


with

$$
\begin{aligned}
& k_{i_{\tau}}=k^{*} \sin \vartheta^{*}=\beta_{i} \sin \xi_{i}+i \alpha_{i} \sin \eta_{i}, \\
& k_{i_{n}}=k^{*} \cos \vartheta^{*}=\beta_{i} \cos \xi_{i}+i \alpha_{i} \cos \eta_{i} ;
\end{aligned}
$$

i.e., we can prefer to divide the wave vector into two components: the tangential one $k_{i_{\tau}}$ and the orthogonal one $k_{i_{n}}$ with respect to the separation interfaces. In order to determine $\beta$ and $\alpha$, we have to impose the well known conditions

$$
\begin{aligned}
\beta^{2}-\alpha^{2} & =k_{\boldsymbol{R}}^{2}-k_{\boldsymbol{I}}^{2}, \\
2 \beta \alpha \cos (\eta-\xi) & =2 k_{\boldsymbol{R}}^{2} k_{\boldsymbol{I}}^{2}
\end{aligned}
$$

[Frezza and Tedeschi 2012]. Solving, we obtain

$$
\begin{aligned}
& \beta=\sqrt{\frac{k_{R}^{2}-k_{I}^{2}}{2}} \sqrt{\sqrt{1+\left[\frac{k_{R} k_{I}}{\left(k_{R}^{2}-k_{I}^{2}\right) \cos \zeta}\right]^{2}}+1}, \\
& \alpha=\sqrt{\frac{k_{R}^{2}-k_{I}^{2}}{2}} \sqrt{\sqrt{1+\left[\frac{k_{R} k_{I}}{\left(k_{R}^{2}-k_{I}^{2}\right) \cos \zeta}\right]^{2}}-1 .}
\end{aligned}
$$

Before proceeding to the study of the reflection and transmission coefficients of the incident field, we want to determine each parameter presented so far; to do that, we can consider the generalized Snell law on the $j$-th interface

$$
\begin{aligned}
\beta_{j} \sin \xi_{j} & =\beta_{j+1} \sin \xi_{j+1}, \\
\alpha_{j} \sin \eta_{j} & =\alpha_{j+1} \sin \eta_{j+1}, \\
\beta_{j+1}^{2}-\alpha_{j+1}^{2} & =k_{\boldsymbol{R}_{j+1}}^{2}-k_{\boldsymbol{I}_{j+1}}^{2}, \\
\beta_{j+1} \alpha_{j+1} \cos \left(\eta_{j+1}-\xi_{j+1}\right) & =2 k_{\boldsymbol{R}_{j+1}}^{2} k_{\boldsymbol{I}_{j+1}}^{2}
\end{aligned}
$$

[Adler et al. 1960]. Using the methodology adopted in [Frezza and Tedeschi 2012], system (23) can be solved, obtaining

$$
\begin{aligned}
& \beta_{j+1}=\sqrt{\frac{\left|k_{i_{j_{\tau}}}\right|^{2}+\left(k_{\boldsymbol{R}_{j+1}}^{2}-k_{\boldsymbol{I}_{j+1}}^{2}\right)+\left|k_{j+1}^{2}-k_{i_{j_{\tau}}}^{2}\right|}{2}}, \\
& \alpha_{j+1}=\sqrt{\frac{\left|k_{i_{j_{\tau}}}\right|^{2}-\left(k_{\boldsymbol{R}_{j+1}}^{2}-k_{\boldsymbol{I}_{j+1}}^{2}\right)+\left|k_{j+1}^{2}-k_{i_{j_{\tau}}}^{2}\right|}{2}}
\end{aligned}
$$


and for the angles

$$
\begin{aligned}
\sin \xi_{j+1} & =\frac{\beta_{j}}{\beta_{j+1}} \sin \xi_{j}, \\
\sin \eta_{j+1} & =\frac{\alpha_{j}}{\alpha_{j+1}} \sin \eta_{j} .
\end{aligned}
$$

Using the generalized Snell law again, we can extend (24) and (25) to the case of stratified media. In particular, the system of equations (23) can be rewritten as

$$
\begin{aligned}
\beta_{1} & =\beta_{N+1} \frac{\sin \xi_{N+1}}{\sin \xi_{1}}, \\
\alpha_{1} & =\alpha_{N+1} \frac{\sin \eta_{N+1}}{\sin \eta_{1}}, \\
\beta_{N+1}^{2}-\alpha_{N+1}^{2} & =k_{\boldsymbol{R}_{N+1}}^{2}-k_{\boldsymbol{I}_{N+1}}^{2}, \\
\beta_{N+1} \alpha_{N+1} \cos \left(\eta_{N+1}-\xi_{N+1}\right) & =2 k_{\boldsymbol{R}_{N+1}}^{2} k_{\boldsymbol{I}_{N+1}}^{2},
\end{aligned}
$$

which once solved gives

$$
\begin{aligned}
& \beta_{N+1}=\sqrt{\frac{\left|k_{i_{\tau}}\right|^{2}+\left(k_{\boldsymbol{R}_{N+1}}^{2}-k_{\boldsymbol{I}_{N+1}}^{2}\right)+\left|k_{N+1}^{2}-k_{i_{1_{\tau}}}^{2}\right|}{2},} \\
& \alpha_{N+1}=\sqrt{\frac{\left|k_{i_{1_{\tau}}}\right|^{2}-\left(k_{\boldsymbol{R}_{N+1}}^{2}-k_{\boldsymbol{I}_{N+1}}^{2}\right)+\left|k_{N+1}^{2}-k_{i_{\tau}}^{2}\right|}{2} .}
\end{aligned}
$$

Now we can see the phase and attenuation constants are independent of the presence of the intermediate layers, as for the case of the lossless scenario. Therefore, the real and the imaginary parts of the complex angle $\vartheta^{*}$ relevant to the last layer may be written as functions of the phase and attenuation vectors:

$$
\begin{aligned}
\cos \vartheta_{\boldsymbol{R}_{N+1}} & =\frac{k_{\boldsymbol{R}_{N+1}} \beta_{N+1} \cos \xi_{N+1}+k_{\boldsymbol{I}_{N+1}} \alpha_{N+1} \cos \eta_{N+1}}{\sqrt{k_{\boldsymbol{R}_{N+1}}^{2} \beta_{N+1}^{2}-k_{\boldsymbol{I}_{N+1}}^{2} \alpha_{N+1}^{2}+2\left(k_{\boldsymbol{R}_{N+1}} k_{\boldsymbol{I}_{N+1}}\right)^{2}}}, \\
\sin \vartheta_{\boldsymbol{R}_{N+1}} & =\frac{k_{\boldsymbol{R}_{N+1}} \beta_{N+1} \sin \xi_{N+1}+k_{\boldsymbol{I}_{N+1}} \alpha_{N+1} \sin \eta_{N+1}}{\sqrt{k_{\boldsymbol{R}_{N+1}}^{2} \beta_{N+1}^{2}-k_{\boldsymbol{I}_{N+1}}^{2} \alpha_{N+1}^{2}+2\left(k_{\boldsymbol{R}_{N+1}} k_{\boldsymbol{I}_{N+1}}\right)^{2}}}, \\
\vartheta_{\boldsymbol{I}_{N+1}} & =\frac{1}{2} \operatorname{atanh}\left(\frac{2 \beta_{N+1} \alpha_{N+1}}{k_{N+1}^{2}}\right) .
\end{aligned}
$$

To determine the value assumed by $\vartheta_{\boldsymbol{R}_{N+1}}$, both (31) and (32) are needed.

We consider a scattering approach to solve the problem; i.e., we take into account all the fields in the different layers and recursively apply the boundary conditions on each interface in order to find the effective reflection and transmission coefficients. Referring to Figure 1, we consider $N+1$ different media, separated 
by $N$ surfaces, each of them identified by the subscript $j$; i.e., we indicate with $\vartheta_{j}, \varphi_{j}, \varepsilon_{j}, \mu_{j}$ and $k_{j}$ the angle of the propagation vector with the $z$-axis, the angle with the $x$-axis of its projection on the $(x, y)$ plane, the relative permittivity, the relative permeability and the wavenumber of the $j$-th medium. Moreover, each layer has thickness $h_{j}$ with the exception of layers 1 and $N+1$, which are two halfspaces. The $j$-th interface separates the $j$-th medium from the $(j+1)$-th medium. Firstly, we consider the parallel (E) polarization; then we will obtain the same result in perpendicular $(\mathrm{H})$ polarization by duality. Our goal is to determine the effective reflection and transmission coefficients for the electric and the magnetic fields $R_{E}^{E}, T_{E}^{E}$ and $R_{H}^{E}, T_{H}^{E}$, respectively, of the structure:

$$
R_{E}^{E}=\frac{E_{r_{1}}^{E}}{E_{i_{1}}^{E}}, \quad T_{E}^{E}=\frac{E_{i_{N+1}}^{E}}{E_{i_{1}}^{E}}, \quad R_{H}^{E}=\frac{H_{r_{1}}^{E}}{H_{i_{1}}^{E}}, \quad T_{H}^{E}=\frac{H_{i_{N+1}}^{E}}{H_{i_{1}}^{E}} .
$$

Looking at the problem from the point of view of multiple reflections on the interfaces between the media, we expect that, in the $j$-th layer, two plane waves propagate: one in the forward direction, with propagation vector $\boldsymbol{k}_{i_{j}}$, that is the superposition of all the secondary reflected waves in the forward direction and the second one in the backward direction, with propagation vector $\boldsymbol{k}_{r_{j}}$, resulting from the superposition of all the secondary reflected waves in the backward direction (see Figure 1). In the last medium, i.e., the $(N+1)$-th layer, there is no backward wave because it is an infinite layer. While the amplitudes of the waves in each layer are our unknowns, the corresponding wave vectors are determined from the Snell law. In fact, similarly to the case of a single dielectric interface, the tangential components of all the wave vectors must be equal to one another: $k_{i_{\tau}}=k_{r_{\tau \tau}}=k_{i_{j+1_{\tau}}}=k_{r_{j+1_{\tau}}}$. From these equalities, we can derive the expressions of the angles $\vartheta_{i_{j}}^{*}$ and $\vartheta_{r_{j}}^{*}$ in each layer. At this point, in order to obtain the coefficients, we have to impose the boundary conditions on each interface. Imposing the continuity of the tangential components of the electric and magnetic field, we obtain

$$
\begin{aligned}
z_{0} \times\left(\boldsymbol{E}_{i_{j}}+\boldsymbol{E}_{r_{j}}-\boldsymbol{E}_{i_{j+1}}-\boldsymbol{E}_{r_{j+1}}\right)=0 & \text { for } z=z_{j}, \\
\boldsymbol{z}_{0} \times\left(\boldsymbol{H}_{i_{j}}+\boldsymbol{H}_{r_{j}}-\boldsymbol{H}_{i_{j+1}}-\boldsymbol{H}_{r_{j+1}}\right)=0 & \text { for } z=z_{j} .
\end{aligned}
$$

The expressions of the electric and magnetic fields for the $j$-th layer are

$$
\begin{aligned}
\boldsymbol{E}_{i_{j}} & =E_{0 i_{j}} \boldsymbol{y}_{0} e^{i\left[k_{i_{j_{\tau}}} x+k_{i_{j_{n}}}\left(z-z_{j}\right)\right]}, \\
\boldsymbol{E}_{r_{j}} & =E_{0 r_{j}} \boldsymbol{y}_{0} e^{i\left[k_{r_{j_{\tau}}} x-k_{r_{j_{n}}}\left(z-z_{j}\right)\right]}, \\
\boldsymbol{H}_{i_{j}} & =\frac{E_{0 i_{j}}}{\omega \mu_{j}}\left(k_{i_{j_{\tau}}} z_{0}-k_{i_{j_{n}}} \boldsymbol{x}_{0}\right) e^{i\left[k_{j_{j_{\tau}}} x+k_{i_{j_{n}}}\left(z-z_{j}\right)\right]}, \\
\boldsymbol{H}_{r_{j}} & =\frac{E_{0 r_{j}}}{\omega \mu_{j}}\left(k_{r_{j_{\tau}}} z_{0}+k_{r_{j_{n}}} \boldsymbol{x}_{0}\right) e^{i\left[k_{r_{j_{\tau}}} x-k_{r_{n}}\left(z-z_{j+1}\right)\right]} .
\end{aligned}
$$


Replacing these expressions in the boundary conditions, we obtain

$$
\left\{\begin{array}{l}
E_{0 i_{j}}+E_{0 r_{j}}=E_{0 i_{j+1}} e^{-i k_{i j+1_{n}}\left(z_{j+1}-z_{j}\right)}+E_{0 r_{j+1}} e^{i k_{i_{j+1}}\left(z_{j+1}-z_{j}\right)}, \\
E_{0 i_{j}}-E_{0 r_{j}}=\zeta_{j, j+1}\left[E_{0 i_{j+1}} e^{-i k_{i_{j+1}}\left(z_{j+1}-z_{j}\right)}-E_{0 r_{j+1}} e^{i k_{i j+1_{n}}\left(z_{j+1}-z_{j}\right)}\right],
\end{array}\right.
$$

having put, for the sake of simplicity, $\zeta_{j, j+1}=\mu_{j} k_{i_{j+1_{n}}} /\left(\mu_{j+1} k_{i_{j_{n}}}\right)$. The linear system (41) can be written in matrix form, yielding

$$
\left[\begin{array}{c}
E_{0 i_{j+1}} \\
E_{0 r_{j+1}}
\end{array}\right]=\left[M_{j}\right]\left[\begin{array}{l}
E_{0 i_{j}} \\
E_{0 r_{j}}
\end{array}\right]
$$

with

$$
\left[M_{j}\right]=\frac{1}{2 \zeta_{j, j+1}}\left[\begin{array}{cc}
\left(1+\zeta_{j, j+1}\right) e^{i \phi_{j+1}} & -\left(1-\zeta_{j, j+1}\right) e^{i \phi_{j+1}} \\
-\left(1-\zeta_{j, j+1}\right) e^{-i \phi_{j+1}} & \left(1+\zeta_{j, j+1}\right) e^{-i \phi_{j+1}}
\end{array}\right],
$$

having put $\phi_{j+1}=k_{i_{j+1_{n}}}\left(z_{j+1}-z_{j}\right)$, the phase difference between two adjacent layers.

We can highlight the term $1+\zeta_{j, j+1}$ :

$$
\left[M_{j}\right]=\frac{1+\zeta_{j, j+1}}{2 \zeta_{j, j+1}}\left[\begin{array}{cc}
e^{i \phi_{j+1}} & -\frac{1-\zeta_{j, j+1}}{1+\zeta_{j, j+1}} e^{i \phi_{j+1}} \\
-\frac{1-\zeta_{j, j+1}}{1+\zeta_{j, j+1}} e^{-i \phi_{j+1}} & e^{-i \phi_{j+1}}
\end{array}\right]
$$

We can note that the term $2 \zeta_{j, j+1} /\left(1+\zeta_{j, j+1}\right)$ is the Fresnel transmission coefficient between the $j$-th and $(j+1)$-th layer $\left(T_{j, j+1}^{E}\right)$ and the term $\left(1-\zeta_{j, j+1}\right) /\left(1+\zeta_{j, j+1}\right)$ is the Fresnel reflection coefficient $\left(R_{j, j+1}^{E}\right)$. So we can rewrite the $\left[M_{j}\right]$ matrix as

$$
\left[M_{j}\right]=\frac{1}{T_{j, j+1}^{E}}\left[\begin{array}{cc}
e^{i \phi_{j+1}} & -R_{j, j+1}^{E} e^{i \phi_{j+1}} \\
-R_{j, j+1}^{E} e^{-i \phi_{j+1}} & e^{-i \phi_{j+1}}
\end{array}\right] .
$$

Now, it is trivial to analyze the transmission through all the layers; in fact, we can write the transmitted field as

$$
\left[\begin{array}{c}
E_{0 i_{N+1}} \\
0
\end{array}\right]=\left[M_{N}\right]\left[\begin{array}{l}
E_{0 i_{N}} \\
E_{0 r_{N}}
\end{array}\right]=\prod_{\ell=1}^{N}\left[M_{\ell}\right]\left[\begin{array}{l}
E_{0 i_{1}} \\
E_{0 r_{1}}
\end{array}\right]=[M]\left[\begin{array}{l}
E_{0 i_{1}} \\
E_{0 r_{1}}
\end{array}\right] .
$$

About the last layer, there is no reflection to obviate this drawback; it is sufficient to place, only for the last layer, $\phi_{N+1}=0$, i.e., consider the last layer to have zero thickness.

Once the overall matrix is obtained, the effective reflection and transmission coefficients of the structure can be found. If we define the transmission matrix as

$$
[M]=\left[\begin{array}{ll}
M_{11} & M_{12} \\
M_{21} & M_{22}
\end{array}\right]
$$


we have

$$
\left\{\begin{array}{l}
M_{11} E_{0 i_{1}}+M_{12} E_{0 r_{1}}=E_{0 i_{N+1}} \\
M_{21} E_{0 i_{1}}+M_{22} E_{0 r_{1}}=0
\end{array}\right.
$$

then the effective coefficients for the parallel polarization are

$$
\begin{aligned}
R_{E}^{E} & =\frac{E_{r_{1}}^{E}}{E_{i_{1}}^{E}}=-\frac{M_{21}}{M_{22}}, \\
T_{E}^{E} & =\frac{E_{i_{N+1}}^{E}}{E_{i_{1}}^{E}}=\frac{M_{11} M_{22}-M_{12} M_{21}}{M_{22}}=\frac{\operatorname{det}[M]}{M_{22}}, \\
R_{H}^{E} & =\frac{H_{r_{1}}^{E}}{H_{i_{1}}^{E}}=\frac{E_{r_{1}}^{E}\left(k_{r_{1}}^{*} / \omega \mu_{1}\right)}{E_{i_{1}}^{E}\left(k_{i_{1}}^{*} / \omega \mu_{1}\right)}=\frac{E_{r_{1}}^{E}}{E_{i_{1}}^{E}}=R_{E}^{E}=-\frac{M_{21}}{M_{22}}, \\
T_{H}^{E} & =\frac{H_{i_{N+1}}^{E}}{H_{i_{1}}^{E}}=\frac{E_{i_{N+1}}^{E}\left(k_{i_{N+1}}^{*} / \omega \mu_{N+1}\right)}{E_{i_{1}}^{E}\left(k_{i_{1}}^{*} / \omega \mu_{1}\right)} \\
& =\frac{\sqrt{\varepsilon_{N+1}} / \sqrt{\mu_{N+1}}}{\sqrt{\varepsilon_{1}} / \sqrt{\mu_{1}}} \frac{E_{i_{N+1}}^{E}}{E_{i_{1}}^{E}}=\frac{Z_{1}^{*}}{Z_{N+1}^{*}} T_{E}^{E}=\frac{Z_{1}^{*}}{Z_{N+1}^{*}} \frac{\operatorname{det}[M]}{M_{22}} .
\end{aligned}
$$

It should be noticed that the matrix $[M]$ that we derived is not the same obtained in the literature [Born and Wolf 1999] but is its inverse: in fact, usually the relation between the transmitted and the incident fields is considered, while we derived the opposite relation.

About the perpendicular polarization, we can find the expression of the coefficients simply exchanging $\zeta_{j, j+1}=\mu_{j} k_{i_{j+1_{n}}} / \mu_{j+1} k_{i_{j_{n}}}$ with $\chi_{j, j+1}=\varepsilon_{j} k_{i_{j+1_{n}}} / \varepsilon_{j+1} k_{i_{j_{n}}}$. It means that the magnetic fields are related by matrices analogous to the ones in (43) but where the parameters $\zeta_{j, j+1}$ must be substituted with $\chi_{j, j+1}$. In this polarization, the transmission matrix assumes the form

$$
\begin{aligned}
{\left[N_{j}\right] } & =\frac{1}{2 \chi_{j, j+1}}\left[\begin{array}{cc}
\left(1+\chi_{j, j+1}\right) e^{i \phi_{j+1}} & -\left(1-\chi_{j, j+1}\right) e^{i \phi_{j+1}} \\
-\left(1-\chi_{j, j+1}\right) e^{-i \phi_{j+1}} & \left(1+\chi_{j, j+1}\right) e^{-i \phi_{j+1}}
\end{array}\right] \\
& =\frac{1}{T_{j, j+1}^{H}}\left[\begin{array}{cc}
e^{i \phi_{j+1}} & -R_{j, j+1}^{H} e^{i \phi_{j+1}} \\
-R_{j, j+1}^{H} e^{-i \phi_{j+1}} & e^{-i \phi_{j+1}}
\end{array}\right],
\end{aligned}
$$

where $T_{j, j+1}^{H}=2 \chi_{j, j+1} /\left(1+\chi_{j, j+1}\right)$ is the transmission coefficient between the $j$-th and $(j+1)$-th layers and the term $R_{j, j+1}^{H}=\left(1-\chi_{j, j+1}\right) /\left(1+\chi_{j, j+1}\right)$ is the reflection coefficient, both in perpendicular polarization. Now the reflection and transmission coefficients of the electric and magnetic fields are

$$
R_{H}^{H}=-\frac{N_{21}}{N_{22}}, \quad T_{H}^{H}=\frac{\operatorname{det}[N]}{N_{22}}, \quad R_{E}^{H}=-\frac{N_{21}}{N_{22}}, \quad T_{E}^{H}=\frac{Z_{N+1}}{Z_{1}} \frac{\operatorname{det}[N]}{N_{22}} .
$$




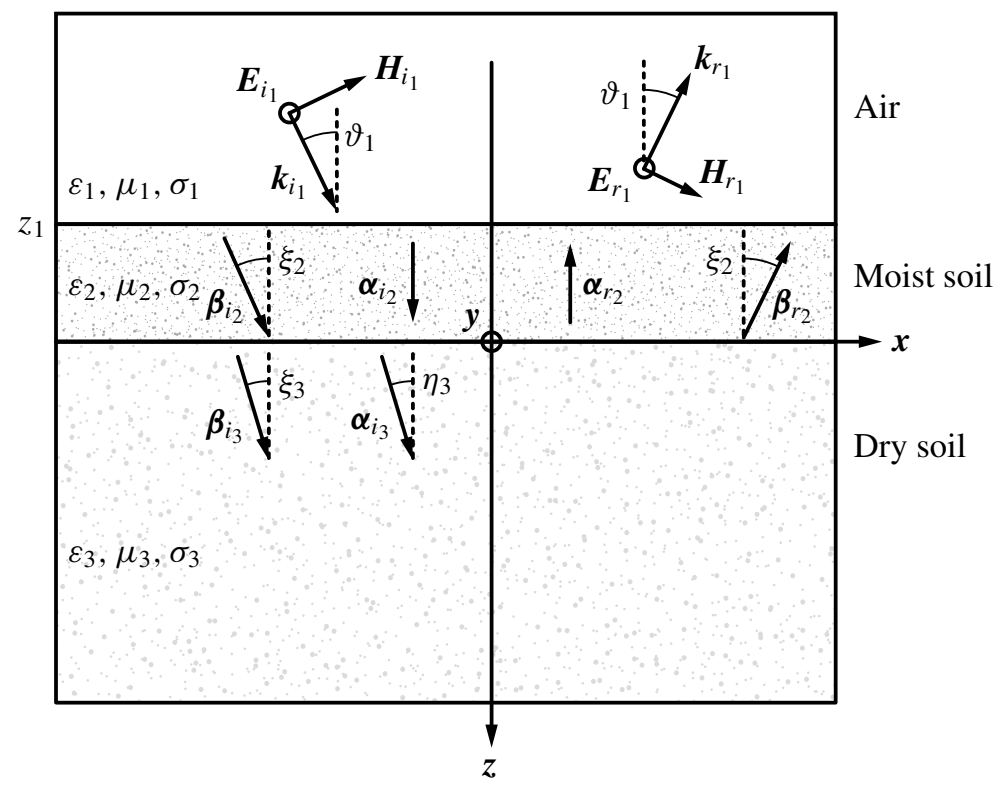

Figure 3. Geometry used to validate the model.

\section{Validation of the model}

To validate our formulation, we compared our results with those of [Curtis 2005], which are based on [Reitz et al. 1979]: their methods exploit an algebraic series that adds up all of the contributions to the net amplitude reflection coefficient of the traveling field in the particular case of just three layers. As a first example, we consider a circular plane wave incident on the stratified medium with the three layers at a frequency of $100 \mathrm{MHz}$ (see Figure 3). The first one is air $\left(\varepsilon_{1}=1\right.$ and $\sigma_{1}=0 \mathrm{~S} / \mathrm{m}$ ), the second one moist soil whose relative dielectric constant is chosen to be $\varepsilon_{2}=10+i 2$, which results in a conductivity of $\sigma_{2}=11.1 \mathrm{mS} / \mathrm{m}$, and the last one dry soil with a complex dielectric constant of $\varepsilon_{3}=3+i 0.2$, which means an effective conductivity of $\sigma_{3}=1.1 \mathrm{mS} / \mathrm{m}$. The thickness of the intermediate layer is $0.05 \mathrm{~m}$ in the first case and $0.20 \mathrm{~m}$ in the second one. In these conditions, we show in Figure 4 the comparison between our results (dashed lines) and the Curtis results (solid line) of the square amplitude of the reflection coefficient in E polarization and $\mathrm{H}$ polarization as a function of the incidence angle: as we can see, the results show a very good agreement in both cases. The second result concerns the square amplitude of the reflection coefficient as a function of the ratio between the toplayer thickness and the top-layer wavelength; the electromagnetic parameters and the geometrical configuration are the same as in the previous case, but now we have considered an incidence angle of 30 degrees (see Figure 5). From the comparisons shown, we can see an optimum agreement, validating our procedure. 

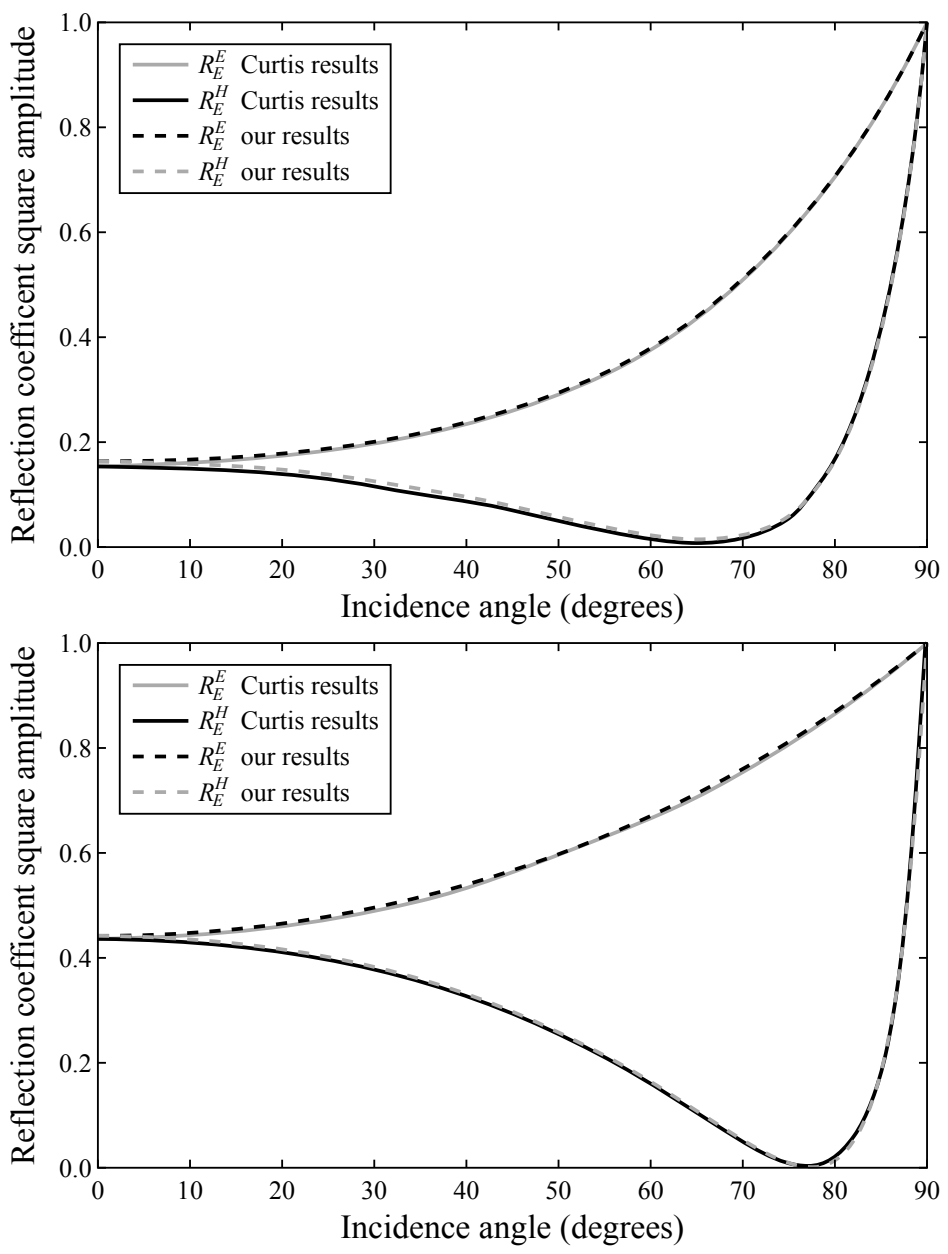

Figure 4. Square amplitude of the reflection coefficient for the electric field as a function of the incidence angle in both polarizations. The stratified medium is located below an air half-space and composed of a layer of moist soil $5 \mathrm{~cm}$ (top) and $20 \mathrm{~cm}$ (bottom) thick on an infinite layer of dry soil.

\section{Conclusion}

In this paper, a rigorous method to solve the electromagnetic scattering problem of an elliptically polarized plane wave by a stratified lossy medium is presented. To determine the reflection and transmission coefficients, we considered the so-called transfer matrix approach. In order to determine each parameter of interest, we have adopted two formalisms: the phase and attenuation vectors and the complex-angle formulation. With these approaches, it is possible to describe all the canonical cases 


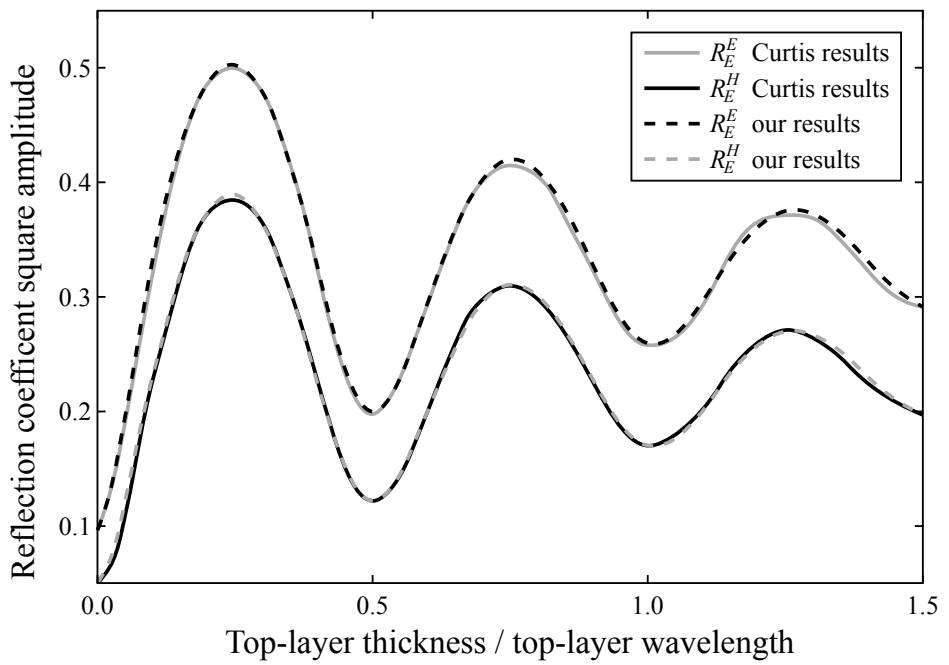

Figure 5. Square amplitude of the reflection coefficient for the electric field as a function of the ratio between the top-layer thickness and the top-layer wavelength in both polarizations with an incident angle of 30 degrees.

in the presence of a multilayered lossy medium, i.e., with or without the presence of a scatterer in the stratified medium. To validate our model, some comparisons with literature results have been presented, obtaining very good agreement in any situation. Obviously this method enjoys the advantages of the transmission-matrix method from which it derives; that is, it can be easily extended to an infinite number of layers to realize an intermediate layer with exotic properties. The generality of the presented method allows its application to several fields of engineering, such as detection of buried or immersed objects, biomedical sensing problems, metamaterial analysis, radar systems, diagnosis of cultural heritage and microscopy; in particular, it can be used to model the lossy medium characterized by a dielectric constant with an anisotropy along the depth direction. Moreover, thanks to this approach, it is possible to design an adaptive material in order to obtain, for example, the zero-reflection and zero-transmission between two external materials: to do this, we can interpose a medium with a relative complex permittivity linearly dependent on the depth.

\section{References}

[Abelès 1950] F. Abelès, "Recherches sur la propagation des ondes électromagnétiques sinusoïdales dans les milieux stratifiés: application aux couches minces”, Ann. Physique (12) 5 (1950), 596-640. [Adler et al. 1960] R. B. Adler, L. J. Chu, and R. M. Fano, Electromagnetic energy transmission and radiation, Wiley, New York, 1960. 
[Besieris 2011] I. M. Besieris, "Comment on the 'Corrected Fresnel coefficients for lossy materials", IEEE Antenn. Propag. M. 53:4 (2011), 161-164.

[Born and Wolf 1999] M. Born and E. Wolf, Principles of optics: electromagnetic theory of propagation, interference and diffraction of light, 7th ed., Cambridge University, 1999.

[Canning 2011] F. X. Canning, "Corrected Fresnel coefficients for lossy materials”, pp. 2123-2126 in 2011 IEEE International Symposium on Antennas and Propagation (Spokane, WA, 2011), IEEE, Piscataway, NJ, 2011.

[Chew 1995] W. C. Chew, Waves and fields in inhomogenous media, IEEE, Piscataway, NJ, 1995.

[Curtis 2005] J. O. Curtis, "A computational tool for simulating plane wave reflectance from layered lossy media", technical report TR-05-3, U.S. Army Corps of Engineers, Engineer Research and Development Center, 2005, Available at http://el.erdc.usace.army.mil/elpubs/pdf/trel05-3.pdf.

[Essinger-Hileman 2013] T. Essinger-Hileman, "Transfer matrix for treating stratified media including birefringent crystals”, Appl. Optics 52:2 (2013), 212-218.

[Feng et al. 2003] Y. Feng, H. Zheng, Z. Zhu, and F. Zu, "The microstructure and electrical conductivity of aluminum alloy foams”, Mater. Chem. Phys. 78:1 (2003), 196-201.

[Frezza and Tedeschi 2012] F. Frezza and N. Tedeschi, "On the electromagnetic power transmission between two lossy media: discussion”, J. Opt. Soc. Am. A 29:11 (2012), 2281-2288.

[Frezza et al. 2013] F. Frezza, F. Mangini, L. Pajewski, G. Schettini, and N. Tedeschi, "Spectral domain method for the electromagnetic scattering by a buried sphere", J. Opt. Soc. Am. A 30:4 (2013), 783-790.

[Frezza et al. 2015] F. Frezza, F. Mangini, and N. Tedeschi, "Electromagnetic scattering by two concentric spheres buried in a stratified material", J. Opt. Soc. Am. A 32:2 (2015), 277-286.

[Ivlev 1987] E. I. Ivlev, "Structure and properties of inhomogeneous waves", J. Mod. Optic. 34:12 (1987), 1559-1569.

[Kaplan and Resnikoff 1967] L. M. Kaplan and M. Resnikoff, "Matrix products and the explicit 3, 6, 9, and 12- $j$ coefficients of the regular representation of $S U(n)$ ", J. Math. Phys. 8:11 (1967), 2194-2205.

[Khoo et al. 2006] I. C. Khoo, D. H. Werner, X. Liang, A. Diaz, and B. Weiner, "Nanosphere dispersed liquid crystals for tunable negative-zero-positive index of refraction in the optical and terahertz regimes", Opt. Lett. 31:17 (2006), 2592-2594.

[Reitz et al. 1979] J. R. Reitz, F. J. Milford, and R. W. Christy, Foundations of electromagnetic theory, 3rd ed., Addison-Wesley, Reading, MA, 1979.

[Roy 2003] J. E. Roy, "New results for the effective propagation constants of nonuniform plane waves at the planar interface of two lossy media", IEEE T. Antenn. Propag. 51:6 (2003), 12061215.

[Takenaka et al. 2003] T. Takenaka, H. Zhou, and T. Tanaka, "Inverse scattering for a three-dimensional object in the time domain", J. Opt. Soc. Am. A 20:10 (2003), 1867-1874.

[Taminiau et al. 2008] T. H. Taminiau, F. D. Stefani, and N. F. van Hulst, "Enhanced directional excitation and emission of single emitters by a nano-optical Yagi-Uda antenna", Opt. Express 16:14 (2008), 10858-10866.

[Vigoureux 1991] J. M. Vigoureux, "Polynomial formulation of reflection and transmission by stratified planar structures", J. Opt. Soc. Am. A 8:11 (1991), 1697-1701.

[Ziolkowski and Erentok 2006] R. W. Ziolkowski and A. Erentok, "Metamaterial-based efficient electrically small antennas”, IEEE T. Antenn. Propag. 54:7 (2006), 2113-2130. 
Received 14 Dec 2015. Revised 17 Feb 2016. Accepted 1 Apr 2016.

FABIO MANGINI: fabio.mangini@uniroma1.it

Information Engineering, Electronics and Telecommunications, Sapienza University of Rome, Via Eudossiana 18, I-00184 Roma, Italy

FABRIZIO FREZZA: fabrizio.frezza@uniroma1.it

Information Engineering, Electronics and Telecommunications, Sapienza University of Rome, Via Eudossiana 18, I-00184 Roma, Italy 
EDITORIAL BOARD

ANTONIO CARCATERRA

ERIC A. CARLEN

FRANCESCO DELL'ISOLA

RAFFAELE ESPOSITO

ALBERT FANNJIANG

Gilles A. FranCFORT

Pierangelo MARCATI

JEAN-JACQUES MARIGO

PETER A. MARKOWICH

MARTIN OSTOJA-STARZEWSKI

PIERRE SEPPECHER

DAVID J. STEIGMANN

PAUl STEINMANN

PierRe M. SuQueT

MANAGING EDITORS

MICOL AMAR

CORRADO LATTANZIO

ANGELA MADEO

MARTIN OSTOJA-STARZEWSKI

ADVISORY BOARD

ADNAN AKAY

Holm AltenBaCH

MICOL AMAR

HARM ASKES

TEODOR ATANACKOVIĆ

VICTOR BERDICHEVSKY

GUY BOUCHITTÉ

ANDREA BRAIDES

ROBERTO CAMASSA

MAURO CARFORE

ERIC DARVE

FELIX DARVE

ANNA DE MASI

GianPiEtro DEL Piero

EMMANUELE Di BENEDETTO

BERNOLD FIEDLER

IRENE M. GAMBA

DAVID Y. GAO

SERGEY GAVRILYUK

TIMOTHY J. HEALEY

DOMINIQUE JEULIN

ROGER E. KHAYAT

CORRADO LATTANZIO

ROBERT P. LIPTON

ANGELO LUONGO

ANGELA MADEO

JUAN J. MANFREDI

CARLO MARCHIORO

GÉRARD A. MAUGIN

ROBERTO NATALINI PATRIZIO NEFF

ANDREY PIATNITSKI

ERRICO PRESUTTI

MARIO PULVIRENTI

LUCIO RUSSO

Miguel A. F. SANJUAN

PATRICK SElVADURAI

ALEXANDER P. SEYRANIAN

MIROSLAV ŠILHAVÝ

GUIDO SWEERS

ANTOINETTE TORDESILLAS

LEV TRUSKINOVSKY

JUAN J. L. VELÁZQUEZ VINCENZO VESPRI ANGELO VULPIANI msp.org/memocs

Università di Roma "La Sapienza", Italia

Rutgers University, USA

(CO-CHAIR) Università di Roma "La Sapienza", Italia

(TREASURER) Università dell'Aquila, Italia

University of California at Davis, USA

(CO-CHAIR) Université Paris-Nord, France

Università dell'Aquila, Italy

École Polytechnique, France

DAMTP Cambridge, UK, and University of Vienna, Austria

(CHAIR MANAGING EDITOR) Univ. of Illinois at Urbana-Champaign, USA

Université du Sud Toulon-Var, France

University of California at Berkeley, USA

Universität Erlangen-Nürnberg, Germany

LMA CNRS Marseille, France

Università di Roma "La Sapienza", Italia

Università dell'Aquila, Italy

Université de Lyon-INSA (Institut National des Sciences Appliquées), France

(CHAIR MANAGING EDITOR) Univ. of Illinois at Urbana-Champaign, USA

Carnegie Mellon University, USA, and Bilkent University, Turkey

Otto-von-Guericke-Universität Magdeburg, Germany

Università di Roma "La Sapienza", Italia

University of Sheffield, UK

University of Novi Sad, Serbia

Wayne State University, USA

Université du Sud Toulon-Var, France

Università di Roma Tor Vergata, Italia

University of North Carolina at Chapel Hill, USA

Università di Pavia, Italia

Stanford University, USA

Institut Polytechnique de Grenoble, France

Università dell'Aquila, Italia

Università di Ferrara and International Research Center MEMOCS, Italia

Vanderbilt University, USA

Freie Universität Berlin, Germany

University of Texas at Austin, USA

Federation University and Australian National University, Australia

Université Aix-Marseille, France

Cornell University, USA

École des Mines, France

University of Western Ontario, Canada

Università dell' Aquila, Italy

Louisiana State University, USA

Università dell'Aquila, Italia

Université de Lyon-INSA (Institut National des Sciences Appliquées), France University of Pittsburgh, USA

Università di Roma "La Sapienza”, Italia

Université Paris VI, France

Istituto per le Applicazioni del Calcolo "M. Picone", Italy

Universität Duisburg-Essen, Germany

Narvik University College, Norway, Russia

Università di Roma Tor Vergata, Italy

Università di Roma "La Sapienza”, Italia

Università di Roma “Tor Vergata”, Italia

Universidad Rey Juan Carlos, Madrid, Spain

McGill University, Canada

Moscow State Lomonosov University, Russia

Academy of Sciences of the Czech Republic

Universität zu Köln, Germany

University of Melbourne, Australia

École Polytechnique, France

Bonn University, Germany

Università di Firenze, Italia

Università di Roma La Sapienza, Italia

MEMOCS (ISSN 2325-3444 electronic, 2326-7186 printed) is a journal of the International Research Center for the Mathematics and Mechanics of Complex Systems at the Università dell'Aquila, Italy.

Cover image: "Tangle” by $\odot$ John Horigan; produced using the Context Free program (contextfreeart.org).

PUBLISHED BY

7 mathematical sciences publishers

nonprofit scientific publishing

http://msp.org/

(C) 2016 Mathematical Sciences Publishers 
Mathematics and Mechanics of Complex Systems vol. 4 no. 2

Constraint reaction and the Peach-Koehler force for dislocation networks

Riccardo Scala and Nicolas Van Goethem

Stability analysis of two coupled oscillators

Mickhail A. Guzev and Alexandr A. Dmitriev

Analysis of the electromagnetic reflection and transmission 153 through a stratified lossy medium of an elliptically

polarized plane wave

Fabio Mangini and Fabrizio Frezza

Dislocation-induced linear-elastic strain dynamics by a

Cahn-Hilliard-type equation

Nicolas Van Goethem

MEMOCS is a journal of the International Research Center for the Mathematics and Mechanics of Complex Systems at the Università dell' Aquila, Italy.

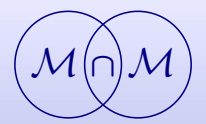

